\title{
MRI Signal Intensity and Electron Ultrastructure Classification Predict the Long-Term Outcome of Skull Base Chordomas
}

\author{
(D). Bai, (D). Shi, (DS. Zhang, (D). Zhang, (D). Zhai, (D). Wang, (D). Li, (DC. Li, (D). Zhao, (D). Geng, \\ (D) S. Gui, (D) L. Jing, and DY. Zhang
}

\begin{abstract}
BACKGROUND AND PURPOSE: MR imaging is a useful and widely used evaluation for chordomas. Prior studies have classified chordomas into cell-dense type and matrix-rich type according to the ultrastructural features. However, the relationship between the MR imaging signal intensity and ultrastructural classification is unknown. We hypothesized that MR imaging signal intensity may predict both tumor ultrastructural classification and prognosis.
\end{abstract}

MATERIALS AND METHODS: Seventy-nine patients with skull base chordomas who underwent 95 operations were included in this retrospective single-center series. Preoperative tumor-to-pons MR imaging signal intensity ratios were calculated and designated as ratio on $T 1$ FLAIR sequence $\left(R_{T 1}\right)$, ratio on $T 2$ sequence $\left(R_{T 2}\right)$, and ratio on enhanced $T 1$ FLAIR sequence $\left(R_{E N}\right)$, respectively. We assessed the relationships among signal intensity ratios, ultrastructural classification, and survival.

RESULTS: Compared with the matrix-rich type group, the cell-dense type chordomas showed lower $\mathrm{R}_{\mathrm{T} 2}$ (cell-dense type: $1.90 \pm$ 0.38; matrix-rich type: $2.61 \pm 0.60 P<.001)$. The model of predicting cell-dense type based on $\mathrm{R}_{\mathrm{T} 2}$ had an area under the curve of 0.83 (95\% Cl, 0.75-0.92). In patients without radiation therapy, both progression-free survival $(P=.003)$ and overall survival $(P=.002)$ were longer in the matrix-rich type group than in the cell-dense type group. $R_{E N}$ was a risk factor for progression-free survival (hazard ratio $=10.24 ; 95 \% \mathrm{Cl}, 1.73-60.79$ ); $\mathrm{R}_{\mathrm{T} 2}$ was a protective factor for overall survival (hazard ratio $=0.33 ; 95 \% \mathrm{Cl}, 0.12-0.87$ ); and $\mathrm{R}_{\mathrm{EN}}$ was a risk factor for overall survival (hazard ratio $=4.76 ; 95 \% \mathrm{Cl}, 1.51-15.01$ ).

CONCLUSIONS: The difference in MR imaging signal intensity in chordomas can be explained by electron microscopic features. Both signal intensity ratios and electron microscopic features may be prognostic factors.

ABBREVIATIONS: $C D T=$ cell-dense type; MRT = matrix-rich type; OS = overall survival; $P F S=$ progression-free survival; $R_{T T}, R_{T 2}$, and $R_{E N}=$ ratios of tumor-to-pons signal intensity in the TT FLAIR sequence, T2 sequence, and enhanced T1 FLAIR sequence, respectively.

C hordoma is a rare malignant tumor with an overall incidence of 0.08 per 100,000 in the United States. ${ }^{1}$ These tumors occur mainly in the sacrum, followed by the skull base and spine. ${ }^{2}$ Skull base chordomas account for $1 \%$ of all brain tumors and $4 \%$ of all primary bone tumors. ${ }^{3}$ Chordomas generally grow slowly; however, recurrence is common because en bloc resection is difficult in the

Received October 1, 2019; accepted after revision March 8, 2020.

From the Department of Neurosurgery (J.B., P.Z., S. Geng, S. Gui, Y. Zhang), Beijing Tiantan Hospital, Capital Medical University, Beijing, China; Beijing Neurosurgical Institute (J.B., S.Z., C.Z., Y. Zhai, S.W., M.L., C.L., Y. Zhang), Capital Medical University, Beijing, China; China National Clinical Research Center for Neurological Diseases (J.B., P.Z., S. Geng, S. Gui, Y. Zhang), Beijing, China; Department of Neurosurgery (I.S.), Tsinghua University Yuquan Hospital, Beijing, China; Department of Neurosurgery (S.Z.), Anshan Central Hospital, Anshan, China; Department of Neurosurgery (Y. Zhai), First Affiliated Hospital, Zhengzhou University, Zhengzhou, China; and Department of Health Statistics (L.J.), Shanxi Medical University, Taiyuan, China.

J. Bai and J. Shi contributed equally to this work.

This work was supported by the Beijing Municipal Science and Technology Commission (No. Z171100000117002) and the Research Special Fund for Public Welfare Industry of Health (No. 201402008). skull base. ${ }^{3-5}$ The recurrence intervals are distinctively variable among patients due to biologic variability among and within patients. This difference also impacts the treatment plan; for example, some patients should receive radiation therapy as early as possible, and others can wait. ${ }^{6}$ Previous studies by our group and others $^{5-12}$ have demonstrated several risk factors related to longterm survival, such as the expression level of Ki-67, Platelet-derived growth factor receptor $\beta$ (PDGFR- $\beta$ ) and SNF5, resection extent, age, postsurgery radiation therapy, and histopathologic findings. On the basis of our previous study on the electron microscopic ultrastructural characteristics of chordomas, we have classified chordomas into 2 different groups: cell-dense type (CDT) and matrix-rich

Please address correspondence to Yazhuo Zhang, MD, Beijing Neurosurgery Institute, Nansihuan Xilu No. 119, Fengtai District, Beijing, 100070, China; e-mail: zyz2004520@yeah.net

- Indicates open access to non-subscribers at www.ajnr.org

http://dx.doi.org/10.3174/ajnr.A6557 


\begin{tabular}{|c|c|c|}
\hline & Cell-Dense Type & Matrix-Rich Type \\
\hline $\begin{array}{l}\text { Tumor cell content in a low-power field } \\
(\times 3000)\end{array}$ & Densely arranged & Seldom or none \\
\hline Extracellular matrix content & Relatively sparse & Abundant \\
\hline $\begin{array}{l}\text { Complex of rough endoplasmic reticulum and } \\
\text { the mitochondria }\end{array}$ & Rich & Relatively seldom \\
\hline Nuclei and chromatin & $\begin{array}{l}\text { Abnormal nuclei with condensed, } \\
\text { deeply staining heterochromatin }\end{array}$ & $\begin{array}{l}\text { Regularly shaped nuclei, mainly with loosely } \\
\text { arranged euchromatin }\end{array}$ \\
\hline Cell junctions & Desmosomes are obvious & Few desmosomes \\
\hline Collagen fibrils & Type III collagen fibrils can be found & Seldom \\
\hline Exocytosis & Easily found & Seldom \\
\hline
\end{tabular}

${ }^{a}$ Reprinted with permission from Bai et $\mathrm{al}^{13}$ and Elsevier.

type (MRT). ${ }^{13}$ We also demonstrated the association between clinical outcomes and electron microscopic features of tumor specimens. ${ }^{13}$ We have shown that CDT is predictive of poorer outcomes, higher risk of recurrence, and shorter survival, though these findings were derived from a small sample size $(n=27)$.

MR imaging allows a differential diagnosis between chordomas and other tumors using signal intensity. ${ }^{14,15}$ Recent studies have suggested that MR imaging signal intensity ratios on different sequences (ratio of tumor-to-pons signal intensity on T1 FLAIR $\left[\mathrm{R}_{\mathrm{T} 1}\right]$; ratio of tumor-to-pons signal intensity on $\mathrm{T} 2\left[\mathrm{R}_{\mathrm{T} 2}\right]$; ratio of tumor-to-pons signal intensity on enhanced T1 FLAIR $\left[\mathrm{R}_{\mathrm{EN}}\right]$ ) are useful in predicting outcomes. Tian et $\mathrm{al}^{16}$ revealed that higher $\mathrm{R}_{\mathrm{T} 2}$ predicted diminished tumor progression and higher $\mathrm{R}_{\mathrm{EN}}$ predicted more rapid tumor progression; this correlation between $\mathrm{R}_{\mathrm{EN}}$ and recurrence or progression was also shown by Lin et $\mathrm{al}^{17}$ However, neither of these studies showed evidence for the possible explanation of signal intensity difference.

We hypothesized that ultrastructural morphologic characters could explain the mechanism of different signal intensities. Hence, this study aimed to explore the relationships among MR imaging signal intensity ratio, the electronic microscopic feature, and survival, which may provide meaningful preoperative prognostic information and help apply better individual-based treatment strategies.

\section{MATERIALS AND METHODS \\ Patients}

Data from 127 patients who underwent an operation by the same surgical team between July 2012 and July 2016 were reviewed retrospectively from the chordoma data base of the neuroendoscopic ward in Beijing Tiantan Hospital. The present series were classified into CDT and MRT according to the electronic features (Table 1). ${ }^{13}$ Overall, in contrast to the features in the MRT tumors, tumor density was high in CDT tumors; thus, the extracellular matrix was sparse. ${ }^{13}$ Only the patients with both electron microscopy images and preoperative MR imaging available were included for further analysis; thus, 79 patients were enrolled in the present study. Among these patients, 27 cases were previously reported. ${ }^{13}$ The relevant clinical information was collected from the inpatient digital archives, including sex, age, tumor status (primary or recurrent tumor), presurgery radiation therapy, tumor blood supply (rich or not rich, with a rich blood supply being defined by having a tumorresection surface that bled easily and was difficult to suction cleanly, while a blood supply was that was not rich was defined by having a tumor resection surface that bled less easily and was easy to suction cleanly), resection rate ( $\geq 90 \%$ or $<90 \%$ ), and histopathology (Ki-67 level, conventional chordoma, or chondroid chordoma). As a retrospective observational study of de-identified data without any additional therapy or monitoring procedures, this study did not need approval from the Ethics Committee of Beijing Tiantan Hospital.

\section{MR Imaging Scans and Image Evaluation}

The patients underwent preoperative MR imaging with a $3 \mathrm{~T} M R$ imaging scanner (Siemens, Erlangen, Germany). Axial unenhanced T1 FLAIR and T2-weighted images were obtained with the following parameters, respectively: TR/TE range $=1850-2500 / 9.4-$ $19.8 \mathrm{~ms}$ and 4500-6000/84-97 ms. Postcontrast scanning was performed 2 minutes after gadopentetate dimeglumine injection, with a concentration of $0.2 \mathrm{~mL} / \mathrm{kg}(0.5 \mathrm{~mol} / \mathrm{L})$. The TR/TE ranges for the enhanced T1 FLAIR sequence were 1850-2500/9.4-19.8 ms. The section thickness was $3.0-50.5 \mathrm{~mm}$.

The images were analyzed on our PACS. Operator-defined ROIs were placed in the tumor and the pons. The mean signal intensity of the tumor was normalized by dividing by the mean signal intensity of the corresponding pons on axial T1 FLAIR $\left(\mathrm{R}_{\mathrm{T} 1}\right)$, $\mathrm{T} 2\left(\mathrm{R}_{\mathrm{T} 2}\right)$, and enhanced T1 FLAIR $\left(\mathrm{R}_{\mathrm{EN}}\right)$, respectively.

\section{Follow-Up}

The follow-up period was calculated from the date of the first operation in Beijing Tiantan Hospital to the last follow-up in March 2019 or the date of death, whichever occurred first. The median followup time in this series was 52 months (range, 1-118 months), and 7 patients were lost to follow-up. Progression-free survival (PFS) was calculated from the date of the operation to the date of tumor recurrence or progression of residual tumor, which was confirmed by the imaging findings; and overall survival (OS) was defined as the time from diagnosis to disease-specific death or to the last follow-up time. Patients were censored at the last follow-up date if no death occurred or on the date of death if death was not disease-specific. ${ }^{5,12}$

\section{Statistical Analysis}

Statistical analyses were performed with SPSS software, Version 24 (IBM). $T$ tests or Mann-Whitney $U$ tests were used to analyze the continuous variables between groups. A $\chi^{2}$ test was used in analyzing the distributions of categoric variables between groups. To evaluate the relationship between signal intensity ratio and electron microscopic classification, we included the signal intensity ratios $\left(\mathrm{R}_{\mathrm{T} 1}, \mathrm{R}_{\mathrm{T} 2}\right.$, $\mathrm{R}_{\mathrm{EN}}$ ) in univariate analyses separately; multivariate analysis was performed using logistic regression. The area under the receiver operating characteristic curve was calculated to estimate the accuracy. 
Table 2: Demographics of the 79 patients undergoing chordoma surgery in Beijing Tiantan Hospital between July 2012 and July 2016

\begin{tabular}{|c|c|c|c|c|}
\hline & Total & MRT & CDT & $P$ \\
\hline No. of cases & 79 & 39 & 40 & \\
\hline \multicolumn{5}{|l|}{ Tumor status } \\
\hline Primary & 37 & 24 & 13 & .01 \\
\hline Recurrent & 42 & 15 & 27 & \\
\hline \multicolumn{5}{|l|}{ Sex } \\
\hline Male & 43 & 21 & 22 & .918 \\
\hline Female & 36 & 18 & 18 & \\
\hline \multicolumn{5}{|l|}{ Age (mean) (yr) } \\
\hline Primary & $47.8 \pm 17.4$ & $42.3 \pm 18.2$ & $58.2 \pm 9.97$ & .006 \\
\hline Recurrent & $47.1 \pm 15.3$ & $46.5 \pm 16.5$ & $47.6 \pm 14.9$ & .828 \\
\hline Total & $47.5 \pm 16.2$ & $43.9 \pm 17.5$ & $51.0 \pm 14.3$ & .050 \\
\hline \multicolumn{5}{|l|}{ Histopathology } \\
\hline Conventional & 71 & 35 & 36 & 1.000 \\
\hline Chondroid & 8 & 4 & 4 & \\
\hline \multicolumn{5}{|l|}{ Blood supply ${ }^{a}$} \\
\hline Rich & 21 & 7 & 14 & .073 \\
\hline Not rich & 55 & 31 & 24 & \\
\hline \multicolumn{5}{|l|}{ Resection grade } \\
\hline$\geq 90 \%$ & 43 & 23 & 20 & .423 \\
\hline$<90 \%$ & 36 & 16 & 20 & \\
\hline \multicolumn{5}{|l|}{ Survival status } \\
\hline Alive & 42 & 27 & 15 & .018 \\
\hline Died & 26 & 8 & 18 & \\
\hline$N A^{b}$ & 11 & 4 & 7 & \\
\hline \multicolumn{5}{|c|}{ Presurgery radiation therapy } \\
\hline Yes & 17 & 5 & 12 & .063 \\
\hline No & 62 & 34 & 28 & \\
\hline \multicolumn{5}{|c|}{ Postsurgery radiation therapy } \\
\hline Yes & 30 & 14 & 16 & .667 \\
\hline No & 43 & 21 & 22 & \\
\hline$N A^{c}$ & 6 & 4 & 2 & \\
\hline PFS $(m o)^{d}$ & $40.3 \pm 5.4$ & $52.2 \pm 5.7$ & $18.7 \pm 6.9$ & .003 \\
\hline OS (mo) & $100.5 \pm 7.1$ & $113.9 \pm 6.3$ & $88.9 \pm 9.3$ & .018 \\
\hline
\end{tabular}

Note:-NA indicates not applicable.

a Operative notes on 3 cases showed no specified blood supply.

${ }^{b}$ Including both patients lost to follow-up and patients who did not die of chordomas.

${ }^{c}$ Including the patients whose radiation therapy information was not available.

${ }^{\mathrm{d}}$ Patients without radiation therapy $(n=32)$.

OS and PFS were estimated using the Kaplan-Meier method, and survival curves for the 2 electron microscopic classifications were compared using the log-rank test. Statistically significant variables in the univariate analyses were included in the multivariate Cox proportional hazards regression model, using forward stepwise variable selection, to find significant predictors of recurrence and survival. A $P$ value $<.05$ was considered statistically significant.

\section{RESULTS}

\section{Clinical Characteristics and Electron Microscopic Classifications}

Seventy-nine cases fit the inclusion criteria and were enrolled in the study (Table 2), which included 37 cases with newly diagnosed primary tumors and 42 cases with recurrent tumors or progression of residual tumors after prior operations. CDT chordomas were more common in recurrent tumors than in primary tumors $(P=.01)$. There were 43 men and 36 women, and the mean age was $47.5 \pm$ 16.2 years. Seventy-one cases were confirmed as conventional chordomas, and 8 were chondroid chordomas. Among them, 47 patients received adjuvant radiation therapy, while the other 32 patients received no radiation therapy. Because no medical treatment was approved for chordoma, no patients received any drug treatment.

According to the electron microscopic classification, ${ }^{13} 39$ cases were classified as MRT and 40 cases were classified as CDT in the first operation. The MRT tumor cells were loosely arranged, the extracellular matrix was abundant, and the tumor cells showed bubble-like vacuoles. CDT tumors displayed relatively tightly arranged tumor cells, a less abundant matrix, and vacuoles not as rich as those in MRT cells (Fig 1). In the patients with primary tumors, patients with MRT tumors were younger than patients with CDT tumors $(42.3 \pm 18.2$ years versus $58.2 \pm 9.97$ years, $P=.006$ ).

Sixty-five patients underwent 1 operation during follow-up, and 14 patients underwent repeat operations due to tumor recurrence or progression of residual tumor. No patients underwent a repeat operation because of an incomplete initial resection. Among those 14 patients, 12 patients underwent 2 operations and 2 patients underwent 3 operations. Therefore, a total of 79 patients with 95 operations were included in the following analysis (Table 3). The median PFS for the patients who underwent a repeat operation was 10.0 months (range, 2-42 months). Among the patients who underwent 2 operations, 6 patients were diagnosed with CDT tumors in both operations, 3 patients were diagnosed with MRT tumors in both operations, 2 patients were diagnosed with a MRT tumor in the first operation and a CDT tumor in the second operation, and 1 patient was diagnosed with a CDT tumor in the first operation and an MRT tumor in the second operation. Among the 2 patients who had 3 operations, one was diagnosed each time with CDT tumors, while the other was diagnosed twice with CDT tumors and once with an MRT tumor.

\section{Relationship between Signal Intensity Ratio and Electron Microscopic Classifications}

Presurgery MR images of 95 operations were pooled and analyzed. The CDT chordomas showed lower signal intensity than MRT chordomas on T2-weighted images (Table 4 and Fig 2). The $\mathrm{R}_{\mathrm{T} 1}$ values of CDT chordomas were higher than those of MRT chordomas. The $\mathrm{R}_{\mathrm{EN}}$ value was also higher in patients with CDT than in those with MRT. When only 37 primary tumors were considered, the difference was still significant (Table 4). 

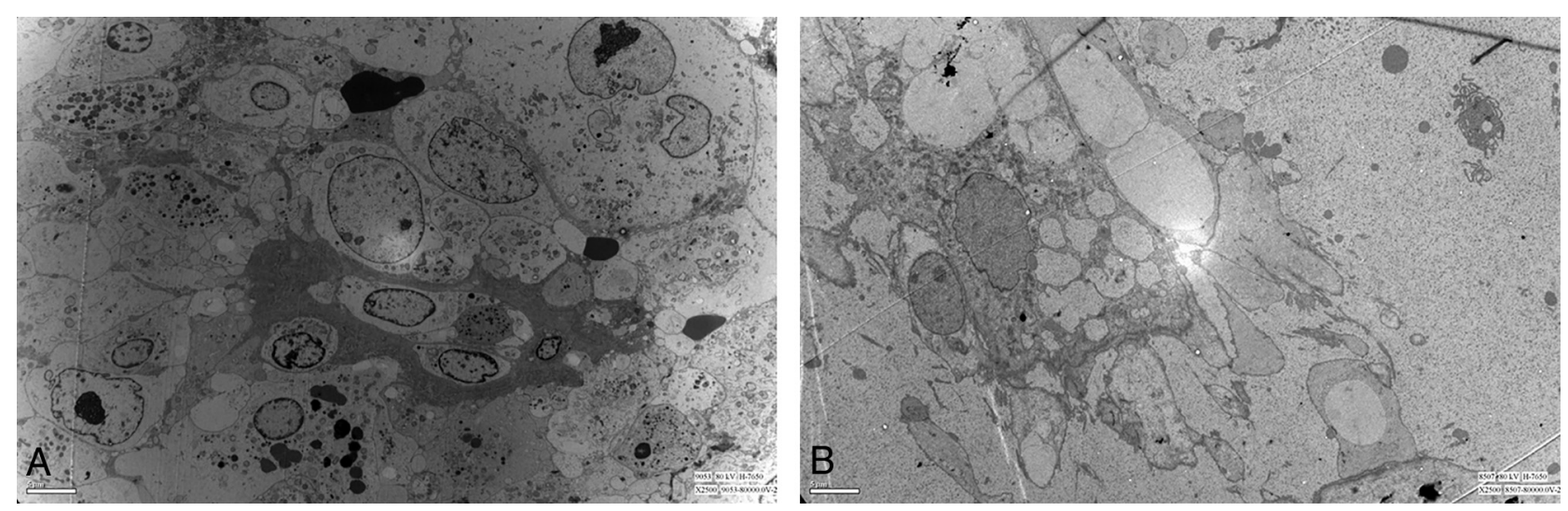

FIG 1. The electron microscopic features of the 2 classifications for skull base chordomas in low-power fields $(\times 2500)$. $A$, In a cell-dense type chordoma, the physaliphorous cells are relatively tightly arranged and there is little extracellular matrix present. The vacuoles are not as rich as those observed in matrix-rich type tumor cells. The nuclei vary in size and condensed nuclei are found. $B$, In a matrix-rich type chordoma, the physaliphorous cells are sparse, and abundant extracellular matrix contents exist. The vacuoles are rich and apparent. The nuclei are slightly atypical.

Table 3: Details for the electron microscopic classification

\begin{tabular}{|c|c|c|}
\hline $\begin{array}{l}\text { Surgery No./Electron Microscopic } \\
\text { Classification }\end{array}$ & $\begin{array}{l}\text { Tumor } \\
\text { Status }^{\mathrm{a}}\end{array}$ & $\begin{array}{l}\text { No. of } \\
\text { Cases }\end{array}$ \\
\hline \multicolumn{3}{|l|}{1 Operation $(n=65)$} \\
\hline \multirow[t]{2}{*}{ CDT } & Primary & 6 \\
\hline & Recurrent & 23 \\
\hline \multirow[t]{2}{*}{ MRT } & Primary & 22 \\
\hline & Recurrent & 14 \\
\hline \multicolumn{3}{|l|}{2 Operations $(n=12)$} \\
\hline \multirow[t]{2}{*}{ Both CDTs } & Primary & 3 \\
\hline & Recurrent & 3 \\
\hline \multirow[t]{2}{*}{ Both MRTs } & Primary & 3 \\
\hline & Recurrent & 0 \\
\hline \multirow[t]{2}{*}{ First MRT, second CDT } & Primary & 1 \\
\hline & Recurrent & 1 \\
\hline \multirow[t]{2}{*}{ First CDT, second MRT } & Primary & 1 \\
\hline & Recurrent & 0 \\
\hline \multicolumn{3}{|l|}{3 Operations $(n=2)$} \\
\hline \multirow[t]{2}{*}{3 CDTs } & Primary & 1 \\
\hline & Recurrent & 0 \\
\hline \multirow[t]{2}{*}{2 CDTs, $1 \mathrm{MRT}^{\mathrm{b}}$} & Primary & 0 \\
\hline & Recurrent & 1 \\
\hline
\end{tabular}

${ }^{\mathrm{a}}$ The tumor status when the first operation was performed in our hospital.

${ }^{\mathrm{b}}$ The second operation was diagnosed as MRT.

Table 4: Comparison of signal intensity ratios between CDT and MRT

\begin{tabular}{|c|c|c|c|}
\hline \multirow[b]{2}{*}{ Signal Intensity Ratio } & \multicolumn{2}{|c|}{$\begin{array}{c}\text { Electron Microscopic } \\
\text { Classification }\end{array}$} & \multirow[b]{2}{*}{$P$} \\
\hline & CDT & MRT & \\
\hline \multicolumn{4}{|l|}{ Entire cohort $(n=95)$} \\
\hline $\mathrm{R}_{\mathrm{T} 2}$ & $1.90 \pm 0.38$ & $2.61 \pm 0.60$ & $<.001$ \\
\hline $\mathrm{R}_{\mathrm{T}}$ & $0.63 \pm 0.14$ & $0.56 \pm 0.13$ & .016 \\
\hline$R_{E N}$ & $1.19 \pm 0.42$ & $0.99 \pm 0.41$ & .022 \\
\hline \multicolumn{4}{|l|}{ Primary tumors $(n=37)$} \\
\hline $\mathrm{R}_{\mathrm{T} 2}$ & $1.99 \pm 0.39$ & $2.69 \pm 0.53$ & $<.001$ \\
\hline $\mathrm{R}_{\mathrm{T}}$ & $0.67 \pm 0.18$ & $0.55 \pm 0.13$ & .019 \\
\hline$R_{E N}$ & $1.17 \pm 0.42$ & $0.89 \pm 0.32$ & .024 \\
\hline \multicolumn{4}{|l|}{ Recurrent tumors $(n=58)$} \\
\hline $\mathrm{R}_{\mathrm{T} 2}$ & $1.87 \pm 0.38$ & $2.50 \pm 0.67$ & .001 \\
\hline $\mathrm{R}_{\mathrm{T}}$ & $0.62 \pm 0.13$ & $0.58 \pm 0.13$ & .307 \\
\hline$R_{E N}$ & $1.19 \pm 0.43$ & $1.11 \pm 0.49$ & .526 \\
\hline
\end{tabular}

However, when only recurrent tumors were analyzed, both $R_{T 1}$ and $\mathrm{R}_{\mathrm{EN}}$ were no longer statistically different, while $\mathrm{R}_{\mathrm{T} 2}$ was still significantly different (Table 4).

Logistic regression analysis was then conducted to predict electron microscopic classification. A univariate analysis indicated that recurrent tumor $(P=.004)$ and decreased $\mathrm{R}_{\mathrm{T} 2}$ $(P<.001)$ and increased $\mathrm{R}_{\mathrm{T} 1}$ and $\mathrm{R}_{\mathrm{EN}}(P=.019$ and 0.027 , respectively) were significant variables for predicting CDT, while presurgery radiation therapy and histopathology subtype were not significant variables $(P>.05)$. From the multivariate analysis, $\mathrm{R}_{\mathrm{T} 2}$ was the only significant independent predictor of CDTs $(P<.001)$. The area under the receiver operating characteristic curve was 0.83 (95\% CI, 0.75-0.92) with only $\mathrm{R}_{\mathrm{T} 2}$ in the model.

\section{Relationship between Electron Microscopic Classifications and PFS and OS}

At follow-up, 26 patients (32.9\%) had died of disease progression. Concerning the 32 patients without radiation therapy, 9 patients had died, including 2 patients in the MRT group and 7 patients in the CDT group. Among the 95 operations, 59 tumors (62.1\%) had recurred.

When all 95 operations were analyzed together, the PFS was significantly longer in the MRT group than in the CDT group, with median PFS values of 36.0 months (interquartile range, 14-73 months) and 10 months (interquartile range, 6-42 months), respectively $(P=.003)$. When the tumors without radiation therapy were considered, the PFS was 56 months (interquartile range, 34-undefined months) in the MRT group and 10 months (interquartile range, 5-24 months) in the CDT group $(P=.003)$ (Fig $3 A)$.

The OS was significantly longer in the MRT group than in the CDT group, with a median OS of 132.0 months (interquartile range, 82-undefined months) and 96.0 months (interquartile range, 48-114 months), respectively ( $n=79$; $P=.018)$. When just the tumors without radiation therapy $(n=32)$ were considered, the MRT group showed a 

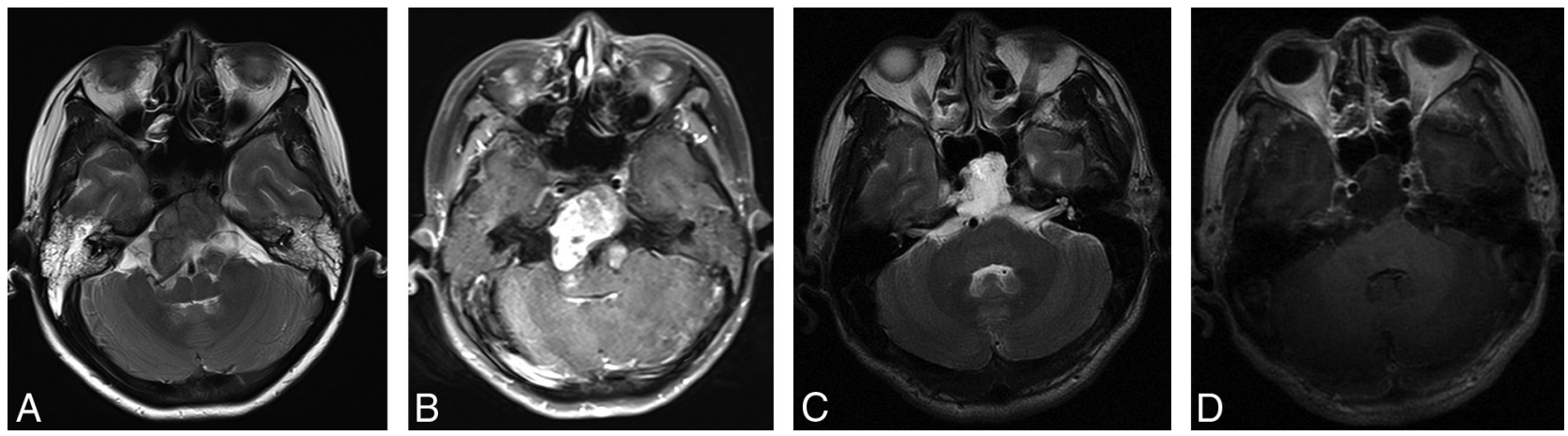

FIG 2. Representative MR images of the 2 classified chordomas. $A$ and $B$, The cell-dense type chordomas. $A$, T2-weighted MR imaging shows a low signal intensity, recurrent chordoma in the clival region. $B$, Contrast-enhanced MR imaging shows the lesion with marked enhancement. $C$ and $D$, Matrix-rich type chordomas with high signal intensity on T2-weighted images and low signal intensity on contrast-enhanced MR imaging, respectively.
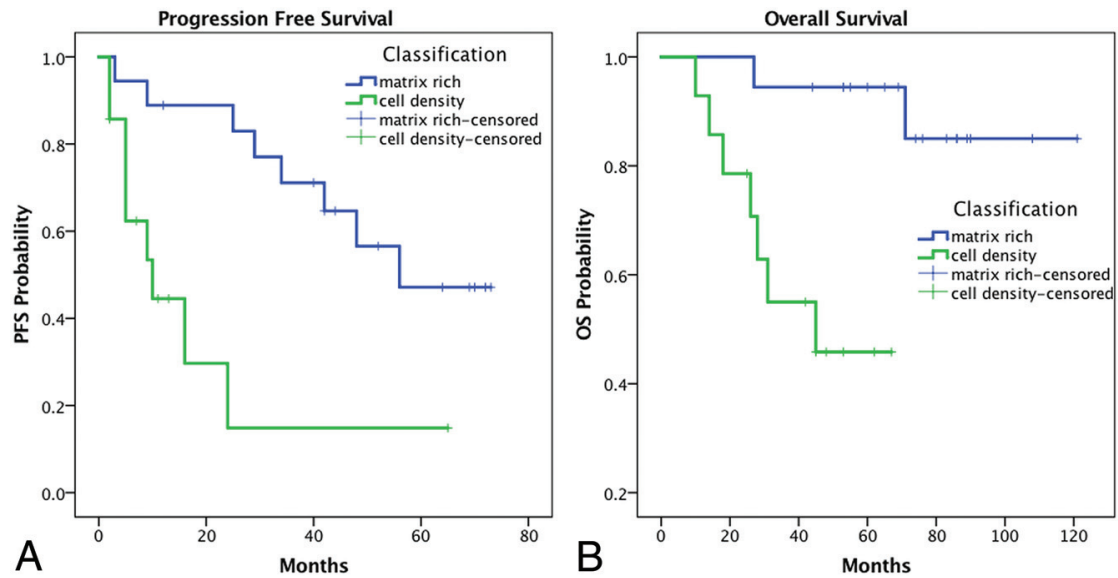

FIG 3. Kaplan-Meier analysis illustrating PFS and OS of 32 patients with chordomas without adjuvant radiation therapy. $A$, The patients with matrix-rich type chordomas have a significantly longer PFS than the patients with cell-dense type chordomas $(P=.003)$. B, The OS of patients with matrix-rich type chordomas is significantly longer than that of those with cell-dense type chordomas $(P=.002)$.

significantly longer OS than the CDT group, with 2 deaths in the MRT group compared with 7 deaths in the CDT group $(P=.002)$ (Fig 3B).

\section{Relationship between MR Imaging Signal Intensity Ratio and PFS and OS}

To test for possible risk factors for recurrence, sex, tumor blood supply, and resection rate were included in the univariate analyses among patients not receiving radiation therapy $(n=32)$. Blood supply and resection rate were associated with recurrence ( $P=.015$ and .019 , respectively). When these 2 parameters were combined with MR imaging signal intensity ratios and age in the multivariate analysis, $\mathrm{R}_{\mathrm{EN}}$ and blood supply were significant risk factors for PFS $\left(\mathrm{R}_{\mathrm{EN}}\right.$ : hazard ratio $=10.24 ; 95 \% \mathrm{CI}, 1.73-60.79$; $P=.010$; blood supply: hazard ratio $=0.87 ; 95 \% \mathrm{CI}, 1.15-13.01$; $P=.029)$.

Univariate Cox regression analyses indicated that postsurgery radiation therapy and resection rate $(P=.023$ and .036 , respectively) were potentially significant prognostic factors for OS in all 79 cases. In the multivariable analysis, $\mathrm{R}_{\mathrm{T} 2}$ and postsurgery radiation therapy were significant protective factors for OS (hazard ratio $=$ 0.33; 95\% CI, 0.12-0.87; $P=.026$; and hazard ratio $=0.12 ; 95 \% \mathrm{CI}, 0.03-$ $0.41 ; P=.001$, respectively). $\mathrm{R}_{\mathrm{EN}}$ was associated with significantly lower OS (hazard ratio $=0.76$; 95\% CI, $1.51-$ 15.01; $P=.008)$.

\section{Relationship between MR Imaging Signal Intensity Ratio and Expression of $\mathrm{Ki}-67$}

The Ki-67 levels were available in 53 samples, which were collected from pathologic reports. The median Ki-67 level was 2\% (range, 0\%-20\%) in 23 MRT chordomas, and the median Ki67 level was $5 \%$ (range, $1 \%-20 \%$ ) in 30 CDT chordomas. The expression of Ki67 was significantly higher in the CDT group than in the MRT group $(P=.033)$.

\section{DISCUSSION}

Skull base chordoma can be an aggressive malignancy with poor prognosis. We previously demonstrated that electron microscopic features of chordomas were variable and could be classified into 2 morphologic types, CDT and MRT. ${ }^{13}$ In the present study, we show that electron microscopic classification is a valid predictor of survival. Patients with CDT had a significantly shorter PFS and OS than those with MRT, even after adjusting for the adjuvant radiation therapy. We further demonstrated that the different electron microscopic features of chordomas exhibited different MR imaging signal intensity ratios, especially $\mathrm{R}_{\mathrm{T} 2}$, and $\mathrm{CDT}$ chordomas had lower $\mathrm{R}_{\mathrm{T} 2}$ than $\mathrm{MRT}$ chordomas. In a recent radiomics study of the differentiation among primary chordoma, giant-cell tumor, and metastatic tumor of the sacrum, the radiomics model constructed on the basis of T2-weighted and contrast-enhanced T1-weighted MR imaging provided good predictive values. ${ }^{18}$ Another radiomics 
study also found that T2-weighted and contrast-enhanced T1weighted MR imaging provided more useful information in differentiating chordoma from chondrosarcoma, compared with T1weighted MR imaging. ${ }^{19}$ An additional study reported that both T2-weighted and contrast-enhanced T1-weighted MR imaging are helpful in differentiating recurrent tumors from postoperative changes. ${ }^{3}$ In the present study, we additionally found that lower $\mathrm{R}_{\mathrm{T} 2}$ was a relatively accurate indicator for CDT (area under the receiver operating characteristic curve $=0.83$; 95\% CI, 0.75-0.92), which is applicable in both primary chordomas and recurrent chordomas.

In CDT chordomas, the tumor cells are rich and contact tightly with each other focally, and the extracellular matrix contents are relatively thin. Conversely, in MRT chordomas, the tumor cells are sparsely arranged and the extracellular matrix contents are abundant. ${ }^{13}$ These morphologic characteristics were also confirmed by hematoxylin-eosin staining under low-power microscopy. ${ }^{20}$ There were numerous vacuoles of various sizes in the cytoplasm of MRT chordomas, while the vacuoles were absent or less obvious in CDT chordomas. Furthermore, the karyoplasm ratio increased occasionally in CDT tumor cells, ${ }^{13}$ which led to reduced fluid content in tumor cells. Both high fluid content in the physaliphorous cells and extracellular matrix likely contribute to the high signal intensity in T2-weighted MR imaging in MRT chordomas. ${ }^{3,14,15}$ Similar findings have been reported in liposarcoma and breast tumors, in which histopathologic components of loose myxoid stroma generate high T2 signal. ${ }^{21,22}$ To our knowledge, this is the first study focusing on the MR imaging signal intensity and electron microscopic features. The results partially-but-directly explain the pathologic basis of the different MR imaging signal intensities among chordomas.

Previous studies ${ }^{15,23}$ demonstrated that poorly differentiated chordomas exhibited no extracellular myxoid stroma or very minimal matrix in pathologic slides and low signal intensity in T2weighted MR imaging, and this subset of patients has decreased survival compared with those with conventional or chondroid chordomas. Although there were no poorly differentiated chordomas in our series, CDT chordomas do have a sparser extracellular matrix and higher tumor cell density, characteristics that make it reasonable to suggest that our results are comparable with those of these studies ${ }^{15,23}$ and that $\mathrm{R}_{\mathrm{T} 2}$ is also qualified in poorly differentiated chordomas.

In the patients who underwent 1 operation by our team, we found a higher ratio of CDT in recurrent tumors $(27 / 42,64.3 \%)$ than in primary tumors $(13 / 37,35.1 \%)$ (Table 2$)$. This finding indicates that recurrent chordomas are more prone to be CDT tumors than primary chordomas. In the patients who underwent $>1$ resection by our team, some tumors recurred as a different electron microscopic type from their previous surgical result. These findings may indicate that the tumor cells were heterogeneously arranged, ${ }^{24}$ and the tiny amount of tissue in the electron microscopic sample may not exactly represent the whole tumor. With MR imaging analysis, the sampling error is greatly reduced because ROIs are selected by drawing the largest possible elliptic area within the tumor but avoiding areas of hemorrhage, necrosis, cyst, or calcification. ${ }^{15,17,25}$ This feature may also indicate the emergence of new malignant cell populations within the recurrent tumor when the MRT tumors recurred as CDT tumors in some cases. Additionally, we found that most of the tumors that underwent multiple resections eventually evolved into CDTs (10/14) (Table 3). A previous study reported that 2 primary sacral chordomas with high signal intensity in T2-weighted images recurred with poorly differentiated chordomas with intermediate signal intensity on T2-weighted images. ${ }^{14}$ This occurrence suggests that decreasing T2 signal intensity may represent increasing malignancy. Recently, we confirmed that Ki-67 was associated with poor prognosis. ${ }^{7}$ In the present study, we found higher Ki-67 levels in CDT tumors than in MRT tumors. The finding suggests that there is a more aggressive proliferation ability in CDT chordomas. ${ }^{13}$ Furthermore, we found that CDT tumors were more likely to recur. ${ }^{13}$ Taken together, we believe that the extent of malignancy increases in most chordomas when they recur, and this progression can be observed by both electron microscopic diagnosis and MR signal intensity.

MR imaging is important not only for diagnosing and differentiating disease but also in predicting prognosis. ${ }^{3,16,24}$ Among the 32 patients who did not undergo adjuvant radiation therapy in the present series, $\mathrm{R}_{\mathrm{EN}}$ and tumor blood supply were demonstrated as independent factors for PFS. Tian et $\mathrm{al}^{16}$ and Lin et $\mathrm{al}^{17}$ have also had similar results, which suggested that $\mathrm{R}_{\mathrm{EN}}$ was a risk factor for PFS. Tian et $\mathrm{al}^{16}$ also found that tumors with higher $\mathrm{R}_{\mathrm{EN}}$ had more tumor blood supply. A recent radiomic analysis of 148 skull base chordomas showed that both radiomic signature (which was based on preoperative axial T1 FLAIR, T2-weighted, and enhanced T1 FLAIR) and blood supply were independent markers of tumor progression. ${ }^{25}$ These 4 independent studies provide convincing evidence to conclude that the high $\mathrm{R}_{\mathrm{EN}}$ and rich blood supply of chordomas can be used as prognostic indicators for rapid recurrence or progression.

We also demonstrated that $\mathrm{R}_{\mathrm{T} 2}$ and postsurgery radiation therapy are protective factors for survival and that $R_{E N}$ is a risk factor for OS. Radiation therapy has been recommended as a standard procedure in chordoma treatment, ${ }^{2}$ and many studies also showed that adjuvant radiation therapy improves outcomes. ${ }^{4,5,26}$ To our knowledge, the correlation between signal intensity ratio and OS in chordomas has not been studied previously, and the precise mechanism of our findings needs further research. However, considering that 79 cases are a relatively large sample size for the low incidence and prevalence of chordoma and the statistical significance of our findings, our results are meaningful. Therefore, MR imaging signal intensity ratios are useful clinical indicators for risk stratification of patients with chordomas or treatment-plan ranking.

\section{Limitations}

The main limitation of this study is that the time interval between the operation and adjuvant radiation therapy was not available in the present data base, and radiation therapy most likely positively influences recurrence intervals. ${ }^{4,5}$ Furthermore, radiation therapy has been shown to impact the MR imaging enhancement parameters. ${ }^{24}$ Therefore, we were unable to control for the effect of radiation therapy in the progression-free survival models. Another limitation is that this is a retrospective study for which some clinical information was missing; for example, the tumor blood supply of 3 tumors was not recorded in the archives. A study showed that lower ADC values may predict tumor 
progression in postsurgery chordomas ${ }^{27}$ and may also predict tumor-control probability in skull base chordomas treated with carbon ion therapy. ${ }^{28}$ This probability suggests that the relationship between ADC values and electronic microscopic characteristics will also be worth investigating. However, diffusion-weighted MR imaging was previously not routinely performed in our hospital; therefore, ADC values were also not available in the present data base. These deficiencies will be remedied in our future study. ${ }^{15}$

\section{CONCLUSIONS}

MR imaging signal intensity ratio could distinguish CDT from MRT preoperatively, particularly $\mathrm{R}_{\mathrm{T} 2}$ values. The electron microscopic features may be the basis of the MR imaging signal intensity difference. Patients with CDT chordomas have significantly shorter PFS and OS than those with MRT chordomas. A higher $\mathrm{R}_{\mathrm{T} 2}$ correlates with an increased likelihood of MRT chordoma and longer survival, while $\mathrm{R}_{\mathrm{EN}}$ is an independent adverse prognostic factor for both PFS and OS.

\section{REFERENCES}

1. McMaster ML, Goldstein AM, Bromley CM, et al. Chordoma: incidence and survival patterns in the United States, 1973. Cancer Causes Control 2001;12:1-11 CrossRef Medline

2. Stacchiotti S, Sommer J; Chordoma Global Consensus Group. Building a global consensus approach to chordoma: a position paper from the medical and patient community. Lancet Oncol 2015;16:e71-83 CrossRef Medline

3. Erdem E, Angtuaco EC, Van Hemert R, et al. Comprehensive review of intracranial chordoma. Radiographics 2003;23:995-1009 CrossRef Medline

4. Walcott BP, Nahed BV, Mohyeldin A, et al. Chordoma: current concepts, management, and future directions. Lancet Oncol 2012;13: e69-76 CrossRef Medline

5. Tauziede-Espariat A, Bresson D, Polivka M, et al. Prognostic and therapeutic markers in chordomas: a study of $\mathbf{2 8 7}$ tumors. J Neuropathol Exp Neurol 2016;75:111-20 CrossRef Medline

6. Beccaria K, Tauziede-Espariat A, Monnien F, et al. Pediatric chordomas: results of a multicentric study of $\mathbf{4 0}$ children and proposal for a histopathological prognostic grading system and new therapeutic strategies. J Neuropathol Exp Neurol 2018;77:207-15 CrossRef Medline

7. Zhang S, Bai J, Li M, et al. Predictive value of transforming growth factor-alpha and Ki-67 for the prognosis of skull base chordoma. World Neurosurg 2019;129:e199-206 CrossRef Medline

8. Zhai Y, Bai J, Wang S, et al. Analysis of clinical factors and PDGFRbeta in predicting prognosis of patients with clival chordoma. $J$ Neurosurg 2018;129:1429-37 CrossRef Medline

9. Li M, Zhai Y, Bai J, et al. SNF5 as a prognostic factor in skull base chordoma. J Neurooncol 2018;137:139-46 CrossRef Medline

10. Zhai Y, Bai J, Wang S, et al. Differences in dural penetration of clival chordomas are associated with different prognosis and expression of platelet-derived growth factor receptor-beta. World Neurosurg 2017;98:288-95 CrossRef Medline
11. Tian K, Wang L, Wang K, et al. Analysis of clinical features and outcomes of skull base chordoma in different age-groups. World Neurosurg 2016;92:407- 17 CrossRef Medline

12. Wang $\mathrm{L}, \mathrm{Wu} \mathrm{Z}$, Tian $\mathrm{K}$, et al. Clinical features and surgical outcomes of patients with skull base chordoma: a retrospective analysis of 238 patients. J Neurosurg 2017;127:1257-67 CrossRef Medline

13. Bai J, Zhai Y, Wang S, et al. Prognostic value of a category based on electron microscopic features of clival chordomas. World Neurosurg 2017;99:282-87 CrossRef Medline

14. Sung MS, Lee GK, Kang HS, et al. Sacrococcygeal chordoma: MR imaging in 30 patients. Skeletal Radiol 2005;34:87-94 CrossRef Medline

15. Yeom KW, Lober RM, Mobley BC, et al. Diffusion-weighted MRI: distinction of skull base chordoma from chondrosarcoma. AJNR Am J Neuroradiol 2013;34:1056-61 CrossRef Medline

16. Tian $\mathrm{K}, \mathrm{W}$ ang $\mathrm{L}, \mathrm{Ma}$ J, et al. MR imaging grading system for skull base chordoma. AJNR Am J Neuroradiol 2017;38:1206-11 CrossRef Medline

17. Lin E, Scognamiglio T, Zhao Y, et al. Prognostic implications of gadolinium enhancement of skull base chordomas. AJNR Am Neuroradiol 2018;39:1509-15 CrossRef Medline

18. Yin $\mathrm{P}, \mathrm{Mao} \mathrm{N}$, Zhao $\mathrm{C}$, et al. A triple-classification radiomics model for the differentiation of primary chordoma, giant cell tumor, and metastatic tumor of sacrum based on T2-weighted and contrastenhanced T1-weighted MRI. J Magn Reson Imaging 2019;49:752-59 CrossRef Medline

19. Li L, Wang K, Ma X, et al. Radiomic analysis of multiparametric magnetic resonance imaging for differentiating skull base chordoma and chondrosarcoma. Eur J Radiol 2019;118:81-87 CrossRef Medline

20. von Witzleben A, Goerttler LT, Lennerz J, et al. In chordoma, metastasis, recurrences, Ki-67 index, and a matrix-poor phenotype are associated with patients' shorter overall survival. Eur Spine J 2016;25:401624 CrossRef Medline

21. Tateishi U, Hasegawa T, Beppu Y, et al. Prognostic significance of MRI findings in patients with myxoid-round cell liposarcoma. AJR Am J Roentgenol 2004;182:725-31 CrossRef Medline

22. Santamaria G, Velasco M, Bargallo X, et al. Radiologic and pathologic findings in breast tumors with high signal intensity on T2-weighted MR images. Radiographics 2010;30:533-48 CrossRef Medline

23. Shih AR, Cote GM, Chebib I, et al. Clinicopathologic characteristics of poorly differentiated chordoma. Mod Pathol 2018;31:1237-45 CrossRef Medline

24. Santos P, Peck KK, Arevalo-Perez J, et al. T1-weighted dynamic contrastenhanced MR perfusion imaging characterizes tumor response to radiation therapy in chordoma. AJNR Am J Neuroradiol 2017;38:2210-16 CrossRef Medline

25. Wei W, Wang K, Liu Z, et al. Radiomic signature: a novel magnetic resonance imaging-based prognostic biomarker in patients with skull base chordoma. Radiother Oncol 2019;141:239-46 CrossRef Medline

26. Zhai Y, Bai J, Wang S, et al. Aberrant expression of extracellular signalregulated kinase and 15-hydroxyprostaglandin dehydrogenase indicates radiation resistance and poor prognosis for patients with clival chordomas. World Neurosurg 2018;115:e146-51 CrossRef Medline

27. Sasaki T, Moritani T, Belay A, et al. Role of the apparent diffusion coefficient as a predictor of tumor progression in patients with chordoma. AJNR Am J Neuroradiol 2018;39:1316-21 CrossRef Medline

28. Buizza G, Molinelli S, D'Ippolito E, et al. MRI-based tumour control probability in skull-base chordomas treated with carbon-ion therapy. Radiother Oncol 2019;137:32-37 CrossRef Medline 